\title{
Research on exacting oil technique of peanut with low temperature
}

\author{
Jie Chen ${ }^{1, a}$, Lihua Zhang', b*, Qing Zhang ${ }^{1, c}$ \\ ${ }^{1}$ Food College, Sichuan Agricultural University, Ya'an, Sichuan, China \\ ${ }^{2}$ College of Mechanical and Electrical Engineering, Sichuan Agricultural University, Ya'an, Sichuan, \\ China \\ $\mathrm{a}, \mathrm{b}, \mathrm{c} 625014$
}

\begin{abstract}
Keywords: peanut; cold-pressing; oil quality; oil yield
Abstract. This research was carried out to investigate the effect of three factors related with cold-pressing technology including pressing temperature, moisture content and shell content on the acid value, moisture and volatile matter content and yield of peanut oil with method of orthogonal experiment of 3 factors and 3 levels. The results showed that moisture content of peanut kernels had the most significant effect on yield and quality of peanut oil, followed by pressing temperature and shell content. When consider the acid value-oriented, the pressing temperature impacted mostly, followed by moisture content and shell content. The oil temperature of $65^{\circ} \mathrm{C}$, moisture content of $7 \%$ and shell content of $21 \%$ were proved to be the optimum technological conditions, under which the acid value of oil was 0.133 , the moisture and volatile matter content of peanut oil was $0.015 \%$, and the oil yield could reach up to $39.8 \%$. The method of squeezing peanut oil in the low temperature is an ideal way to produce peanut oil due to the fact that the physical and chemical indicators of oil meet the national standard, and even better than the national standard.
\end{abstract}

\section{Introduction}

Peanut (Arachis hypogaea) is one of the commonly cultivated legume crops, containing about $24 \%$ $36 \%$ of protein and eight kinds of essential amino acids which the digestion rate could reach up to $99 \%$. Besides peanut is cholesterol-free, leading it be an important food in maintaining the infant's nutritional requirement and human health[1,2]. Peanut oil is one of the processed products which can be used as the raw material of edible oil, health care products and medicine [3-6]. The economic benefits and social benefits of peanut oil are obvious.

The main extraction method of peanut oil is physical squeezing, including hot-pressing technology and cold-pressing technology. When compared with hot-pressing technology, oil produced by cold-pressing technology preserves more bioactive substances, such as vitamin E and sterol etc. [7]. The quality of peanut oil produced by cold-press is better for the reasons of more clear appearance, higher linoleic acid content, lower low acid value and peroxide value as compared with the oil from hot-pressing [8-10]. At the same time, the meal from cold-pressing technology possesses higher nutritional value. Based on above benefits, cold-pressing technology is an ideal method for oil extraction. Even so, cold-pressing technology still has some problems, for instance low oil yield and high residual oil rate which need to be investigated further $[11,12]$.

The objectives of this research were to investigate the effect of factors of cold-pressing technology including pressing temperature, moisture content and shell content on the acid value, moisture and volatile matter content and yield of peanut oil. At last, the main factors affecting the yield and the optimum parameters of cold-pressed peanut oil were determined in order to provide some references for improving the oil yield of cold-pressing technology.

\section{Materials and methods}

Materials and Reagents. Peanuts were obtained from Guangtong Grain and Oil Co. Ltd. (Chengdu, Sichuan Province, China). Anhydrous ether, petroleum ether, potassium hydroxide, sodium hydroxide, acetone, hydrochloric acid, anhydrous sodium sulfate were purchased from the Kelong Chemical Reagent Factory. (Chengdu, China), and they were all analytical grade. 
Main instruments and equipments. Automatic frying combined press (FY172-C, Er Long grain and oil equipment Co. Ltd. Shanghai, China).Peanut sheller (12-40A,Rong Long Co. Ltd, Henan, China). Centrifugal filter(YTLY-80, Yuantong Machinery Equipment Co. Ltd, Henan, China). Oven(DHG-9101-3SA, Nanbei Instrument and Equipment Co. Ltd, Henan, China)

Technological process. The peanut was put into the press for cold pressing after shelling, crushing, regulation of moisture content and frying. Thereafter, the peanut oil was filtered.

Experimental design. According to the results of single factor experiment, orthogonal experiment with 3 factors (pressing temperature, moisture content and shell content) and 3 levels of each factor was carried out. The acid value, moisture and volatile matter content and oil yield of peanut were as targets. The factors and levels of each factor were shown in table 1.

Table 1 Factors and levels of orthogonal experiment

\begin{tabular}{cccc}
\hline & A & B & C \\
level & Pressing temperature $\left({ }^{\circ} \mathrm{C}\right)$ & Moisture content $(\%)$ & Shell content (\%) \\
\hline 1 & 45 & 4 & 7 \\
2 & 55 & 7 & 14 \\
3 & 65 & 10 & 21
\end{tabular}

Determination of moisture content. The moisture content in peanut kernel was determined with the constant weight method[13].

Determination of acid value in Peanut oil. The acid value of peanut oil was test according to the methods of titration with some modifications [14]. The acid value were calculated using the following formulas, Eq. 1

$$
S=\frac{56.1 \bullet V \bullet c}{m} .
$$

Where $\mathrm{S}(\mathrm{mg} \mathrm{KOH} / \mathrm{g})$ is the acid value of peanut oil, $\mathrm{V}(\mathrm{mL})$ is the volume of the standard volumetric potassium hydroxide solution, $\mathrm{C}(\mathrm{mol} / \mathrm{L})$ is the accurate concentration of the standard volumetric potassium hydroxide solution, $56.1(\mathrm{~g} / \mathrm{mol})$ is the molar mass of the potassium hydroxide.

Determination of moisture and volatile matter content of peanut oil. According to the method which Animal and vegetable fats and oils-Determination of moisture and volatile matter content to determination [15]. They were calculated using the following formulas, Eq. 2

$$
X=\frac{m_{1}-m_{2}}{m_{1}-m_{0}} \times 100 \%
$$

Where $\mathrm{X}(\%)$ is the moisture and volatile matter content of peanut oil, $\mathrm{m}_{0}(\mathrm{~g})$ is the quality of glass container, $\mathrm{m}_{1}(\mathrm{~g})$ is the quality of glass container and test sample before heating, $\mathrm{m}_{2}(\mathrm{~g})$ is the the quality of glass container and test sample after heating.

Determination of oil yield of peanut. The cold pressed peanut oil will be made of centrifuged and filtered, then weighing, calculating the oil yield which were calculated using the following formulas, Eq. 3

$$
R=\frac{m_{1}}{m} \times 100 \%
$$

Where $\mathrm{R}(\%)$ is the oil yield of peanut, $\mathrm{m}_{1}(\mathrm{~g})$ is the oil output, $\mathrm{m}(\mathrm{g})$ is the weight of the oilseed. 


\section{Results and discussion}

The experimental results which analyzed by the method of range analysis [16] and comprehensive balance were shown in table 2 and table 3. According to the column of oil yield, moisture and volatile matter content in Table 3, moisture content had most important impact on oil yield and volatile matter content of peanut oil from cold-pressing technology, followed by pressing temperature and shell content. Possible reason may be moisture in material will affect the physical properties of oilseed, such as thermal conductivity, mechanical strength and plasticity etc, among which plasticity had the greatest influence on the squeezing effect, and the plasticity is mainly affected by moisture and temperature. However, the temperature is low in cold-pressing technology, so we mainly change plasticity by adjusting the moisture [17]. In terms of the target of oil yield, the optimal process condition for cold-pressing peanut oil is the combination of $\mathrm{A}_{3} \mathrm{~B}_{2} \mathrm{C}_{3}$. In general, the moisture in oilseed won't be completely evaporated in the roasting process, those moisture will be mixed in the oil. In terms of the target of moisture and volatile matter content, the optimal process condition of cold-press peanut oil is the combination of $\mathrm{A}_{3} \mathrm{~B}_{1} \mathrm{C}_{1}$.

According to the column of acid value of Table 3, the pressing temperature impacted the oil yield of peanut oil mostly, followed by moisture content and shell content. The acid value of oil is related to raw material, production and processing technology of oil, and so on. As the raw materials of different combinations were the same in this experiment, so we main consider the effect of preparing and processing technology. In the process of producing oil, triglycerides in oil under the action of heat and the lipolytic enzyme to decompose of free fatty acids, which increase the acid value. In terms of the target of acid value, the optimal process condition of cold-press peanut oil is the combination of $\mathrm{A}_{1} \mathrm{~B}_{3} \mathrm{C}_{3}$.

As the above analysis showed, the optimal combination from the analysis of the three indexes are inconsistent, so the results need to be made comprehensive balance with integrated balance method according to the primary and secondary relation, the optimal level is $\mathrm{A}_{3} \mathrm{~B}_{2} \mathrm{C}_{3}$, by which the oil yield was as high as $39.8 \%$ with the acid value and moisture and volatile matter content and so on are in line with national standards.

Table 2 Results of orthogonal test

\begin{tabular}{ccccccc}
\hline test number & A & B & C & oil yield $(\%)$ & $\begin{array}{c}\text { Acid value }(\mathrm{mg} \\
\mathrm{KOH} / \mathrm{g})\end{array}$ & $\begin{array}{c}\text { moisture and } \\
\text { volatile matter }\end{array}$ \\
\hline 1 & 1 & 1 & 1 & 20.40 & 0.0999 & 0.0163 \\
2 & 1 & 2 & 2 & 35.40 & 0.1053 & 0.0185 \\
3 & 1 & 3 & 3 & 32.60 & 0.0997 & 0.0240 \\
4 & 2 & 1 & 2 & 30.80 & 0.1059 & 0.0184 \\
5 & 2 & 2 & 3 & 35.80 & 0.1159 & 0.0196 \\
6 & 2 & 3 & 1 & 38.0 & 0.1110 & 0.0247 \\
7 & 3 & 1 & 3 & 34.80 & 0.1161 & 0.0143 \\
8 & 3 & 2 & 1 & 35.60 & 0.1559 & 0.0146 \\
9 & 3 & 3 & 2 & 35.80 & 0.1325 & 0.0200 \\
\hline
\end{tabular}


Table 3 Analysis of orthogonal array test results

\begin{tabular}{cccccccccc}
\hline & \multicolumn{3}{c}{ Oil yield/\% } & \multicolumn{3}{c}{ Acid value/(mg KOH/g) } & \multicolumn{3}{c}{ moisture and volatile matter } \\
content $/ \%$
\end{tabular}

Physical and chemical properties of peanut oil. The cold-pressed peanut oil was compared with the national standard for pressed peanut oil. The results are shown in table 4.

Table 4 Physical and chemical properties of cold pressed peanut oil

\begin{tabular}{ccc}
\hline Parameters & national standard & Cold-pressed oil sample \\
\hline acid value $(\mathrm{mg} \mathrm{KOH} / \mathrm{g})$ & $\leq 2.5$ & 0.133 \\
saponified matter content $/ \%$ & $\leq 0.03$ & 0.0005 \\
moisture and volatile matter content $/ \%$ & $\leq 0.15$ & 0.015 \\
odor and taste & normal, no peculiar smell & normal, no peculiar smell \\
transparency & clear and transparent & clear and transparent \\
Color & $\mathrm{Y} \leq 25 ; \mathrm{R} \leq 4.0$ & $\mathrm{Y}=15 ; \mathrm{R}=1.0$ \\
\hline
\end{tabular}

As shown in Table 4, the cold-pressed peanut oil had a good quality with no special odor and taste, the oil was clear and transparent. Apart from this, the acid value, saponified matter content, and color were much lower than these of national standard.

\section{Conclusions}

This research showed the moisture content impacted the peanut oil yield most markedly, followed by pressing temperature and shell content which had the same effect on oil moisture and volatile matter content of peanut oil. However, under the consideration of acid value-oriented, the pressing temperature affect most significantly, followed by moisture content and shell content. Under the premise in ensuring the quality of peanut oil, the oil temperature of $65^{\circ} \mathrm{C}$, moisture content of $7 \%$ and shell content of $21 \%$ were proved to be the optimum technological conditions, under which the acid value of oil was 0.133 , the moisture and volatile matter content was $0.015 \%$, and the oil yield was as high as $39.8 \%$. The method of squeezing peanut oil in the low temperature is the ideal way to produce peanut oil, which the oil yield is high, and the physical and chemical indicators of oil are in line with national standards and even better than the national standards, such as acid value, saponified matter content, moisture and volatile matter content, transparency, taste and odor and so on.

\section{Acknowledgements}

This work was financially supported by Special technical system of modern agricultural industry (No. CARS-04-PS19).

\section{References}

[1] P Zhao, Y Wang, Y Zhang: The extraction of different proteins in selenium enriched peanuts and their antioxidant properties. Saudi Journal of Biological Sciences. 23. 353-357 (2016), p. 3

[2] Jianmei Yu, Mohamed Ahmedna, Ipek Goktepe: Peanut protein concentrate: Production and functional properties as affected by processing. Food Chemistry. 103.121-129 (2007), p.1 
[3] Shao Bing Zhang: In vitro antithrombotic activities of peanut protein hydrolysates. Food Chemistry. 202. 1-8 (2016), p.1

[4] EK Vassiliou, A Gonzalez, C Garcia: Oleic acid and peanut oil high in oleic acid reverse the inhibitory effect of insulin production of the inflammatory cytokine TNF- $\alpha$ both in vitro and in vivo systems. Lipids in Health \& Disease.8.25-26(2009), p.1

[5] RR Bansode, P Randolph, M Ahmedna : Bioavailability of polyphenols from peanut skin extract associated with plasma lipid lowering function. Food Chemistry . 148. 24-29(2014), p.3

[6] Qiuyan Xu, Zhiwei Zhao, Yongmei Zhao,et al:Effects of Peanut Oil on Behavior and Metabolism Rats with Type 2 Diabetic Mellitus. Journal of Capital University of Medical Sciences. 27. 631-633 (2006), p.5

[7] Zhang cun Wang, Yanling Kang: The progress on study of peanut oil production. Science and Technology of Cereals. 15. 40-41 (2007), p.6

[8] Yulan Liu, Ruihua Liu, Xueling Zhong et al: Differences of quality indexes of peanut oil obtained with different processes. China Oils and Fats. 37. 6-10 (2012), p.9

[9] Jingna Liu, Yawei Wang, Bin Wang: Quality changes of cold and hot pressed peanut crude oil during normal storage. China Brewing. 33. 112-114 (2014), p.8

[10] Yang Wang: A comparative study on the properties of cold-pressed and hot-pressed peanut oil. Modern Agriculture.5. 6-8 (2015), p.17

[11] C Soto, JCE Zuniga: Antioxidant content of oil and defatted meal obtained from borage seeds by an enzymatic-aided cold pressing process. Process biochemistry. 43. 696-699 (2008),p.6

[12] Pawel Gornas, Aleksander Siger, Karina Juhnsevica: Cold-pressed Japanese quince (Chaenomeles japonica(Thunb.) Lindl. ex Spach) seed oil as a rich source of a-tocopherol, carotenoids and phenolics: A comparison of the composition and antioxidant activity with nine other plant oils. Lipid Science and Technology. 116.563-570(2014), p.5

[13] Zhou Zhou, Hong Li, Wei He: An improved method for the determination of water content in soybean . Journal of Anhui Agri. Sci. 31. 19594-19595(2011), p39

[14] M Wroniak, K Krygier, M Kaczmarczyk: Comparison of the quality of cold pressed and virgin rapeseed oils with industrially obtained oils. Polish Journal of Food \& Nutrition Sciences. 20. 85-89(2008), p58

[15] Minghui Zhu, Xin Wen, Jinhong Zhao: Effect of Industrial Chemical Refining on the Physicochemical Properties and the Bioactive Minor Components of Peanut Oil. Journal of the American Oil Chemists' Society February. 2. 285-294(2016), p93

[16] QinDe Wang, Jan Yang, in: Food experimental design and statistical analysis, , edited by QD Wang, volume 4 of Statistical analysis of orthogonal test results, chapter, 2,China Agricultural University press (2010)

[17] Yadong Wang, Shaohua Liang, Le Peng: Optimization of conditioning parameters of cold-pressed sesame seed oil by response surface methodology. China Oils and Fats.37. 13-17 (2012), p.6 